\title{
A Class of the Spraying Operation Trajectory Planning and Optimization
}

\author{
Xiao Wei, Songfeng Pan, Ping Wei \\ (The College of Automation and Engineering, Qingdao University, Qingdao 266071,China) \\ 498531650@qq.com
}

\begin{abstract}
Key words: automatic paint spraying system; spray gun; trajectory planning
Abstract. This paper studies the trajectory planning and the optimization of the end spray gun of the mechanical arm, aiming at the running distance of the spray gun. The proposed way makes the whole process of gun required to run the shortest distance of the spraying trajectory. It builds mathematical model towards the optimal trajectory planning of the spray gun's spraying work; according to the process requirements, kinematic parameter are gained by the technological process and the control instruction. It allows the spray gun at the end of the mechanical arm to run as per the expected trajectory by using the trajectory of the spray gun. Based on the approach, it can get a set of automatic spraying device and complete the spraying work with high quality. This study has lots of practical application value.
\end{abstract}

\section{Introduction}

According to the technological requirement of spraying, this project designed and optimized the trajectory of the end executor of the mechanical arm, and determined the position and pose to the nozzle of the spray gun at the end of the mechanical arm on the basis of the optimized trajectory. Reach the values of joint variables on the mechanical arm at this pose through solving inverse kinematics, making the spray gun operate as per the expected trajectory[1]. This paper mainly researched on the optimal trajectory planning and the optimization to the spray gun's spraying work.

\section{The principle of the end executor on the mechanical arm}

In the field of painting industry, the spray gun is an essential production tool, and has many models and types, which are suitable for the spraying requirements in various situations. In this project, it selects the automatic static spray gun as the spraying device. The Electrostatic spray gun uses a new technology that utilizes the electrostatic fields caused by a high voltage to spray paint. This spraying gun's range is from $350 \mathrm{~mm}$ to $400 \mathrm{~mm}$. The mist spray that machined by the spray gun on the work-piece surface is a form approximate cone shape, as is shown in fig. 1. When the spraying gun is working, we should keep it and the work-piece vertical in the machine, meanwhile keep the distance between them constantly and keep the spray gun moving in a constant speed, and bring the overlap of the two adjacent spraying trajectories into correspondence.

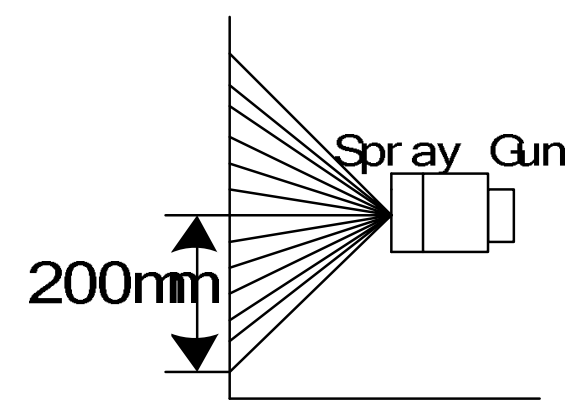

Fig. 1: The Spraying Range Schematic Diagram of the Spray Gun 


\section{The trajectory planning and the optimization of the spray gun's spraying work}

The trajectory planning of the spray gun's spraying work. The key to sprayed the work-piece is to make trajectory planning to the spray gun, which at the end of the arm, aiming that to choose the initial point and processing sequence, and the operation trajectory of the gun, according to the requirement of the spraying work. Suppose the work-piece to be machined is the stationary one with square surface, as shown in fig. 2, then the surface of the work-piece to be machined could be expressed as:

$$
S=\left\{(x, y), x_{1} \leq x \leq x_{2}, y_{1} \leq y \leq y_{2}\right\}
$$

Adopt Cartesian coordinate system XYZ to describe the form and the position of the work-piece to be machined. The function formula of work-piece surface is defined as:

$$
S=\{(x, y, z), z=h(x, y),(x, y) \in D\}
$$

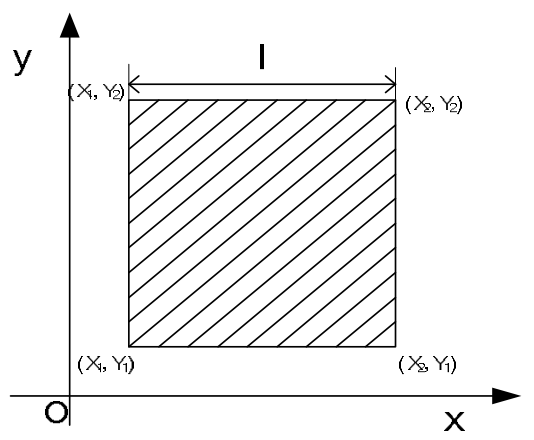

Fig. 2: The Model of the Work-piece that to be Machined

As the surface of the work-piece to be machined is plane, when carry out the spraying work, the spray gun and the surface of the work-piece (set the distance between the spray gun and the surface of the work-piece is h), always keep vertical and equal distance. In fact, the spray gun moves on the isometric face of the work-piece surface. Then the space trajectory of the spray gun is:

$$
a(t)=\left[\begin{array}{llllll}
a_{x}(t) & a_{y}(t) & h & 0 & 0 & 0
\end{array}\right]
$$

Suppose the relative position of the spray gun to the Cartesian coordinate system $\mathrm{XYZ}$ is expressed a three-dimensional vector function $\mathrm{p}(\mathrm{t})$, the relative direction of the spray gun to the Cartesian coordinate system $\mathrm{XYZ}$ is expressed as three dimensional vector $\mathrm{o}(\mathrm{t})$, then both vector functions could be expressed as:

$$
\begin{aligned}
& p(\mathrm{t})=\left[\begin{array}{lll}
p_{x}(t) & p_{y}(t) & p_{z}(t)
\end{array}\right] \\
& o(t)=\left[\begin{array}{lll}
o_{x}(t) & o_{y}(t) & o_{z}(t)
\end{array}\right]
\end{aligned}
$$

In these formulas, $\mathrm{p}(\mathrm{t})$ indicates the relative position of the spray gun to the Cartesian coordinate system, $\mathrm{o}(\mathrm{t})$ indicates the relative rotation angle of the spray gun to the Cartesian coordinate system. Both of them are the functions of $t$. By using the vector function a(t) as the direction and position of the spray gun, the formula is expressed as below:

$$
a(t)=[p(t) o(t)]
$$

Set "a" as the spraying brand width of the spray gun, the motion trajectory of the spraying work has the following forms:

1) The spray gun trajectory operation of a work-piece to be processed from a point $\left(x_{1}, y_{1}+a / 2\right)$ on one side of the work-piece linear motion to a point $\left(\mathrm{x}_{2}, \mathrm{y}_{1}+\mathrm{a} / 2\right)$ on the other side, make the spray gun go back to the starting position, and then move a point $\left(x_{1}, y_{1}+3 a / 2\right)$ on one side of the 
work-piece to a point $\left(\mathrm{x}_{2}, \mathrm{y}_{1}+3 \mathrm{a} / 2\right)$ on the other side of the work-piece, which is as shown in table 1 . According to the periodic course of this path, the distance between two adjacent paths is equal[2]. The path of the spray gun was shown in fig. 3 . The total length of this path is: $L=2 \times n \times 1+n \times a$, in this formula, $\mathrm{n}$ is the spraying journey, the distance between the adjacent spraying paths is a.

Table 1 The Motion Trajectory of the Spray Gun

\begin{tabular}{|c|c|c|}
\hline Spray Path & Starting point & Termination point \\
\hline 1 & $\left(x_{1}, y_{1}+a / 2\right)$ & $\left(x_{2}, y_{1}+a / 2\right)$ \\
\hline 2 & $\left(x_{1}, y_{1}+3 a / 2\right)$ & $\left(x_{2}, y_{1}+3 a / 2\right)$ \\
\hline 3 & $\left(\mathrm{x}_{1}, \mathrm{y}_{1}+5 \mathrm{a} / 2\right)$ & $\left(x_{2}, y_{1}+5 a / 2\right)$ \\
\hline$\ldots \ldots$ & $\ldots \ldots$ & $\ldots \ldots$ \\
\hline $\mathrm{n}$ & {$\left[\mathrm{x}_{1}, \mathrm{y}_{1}+\mathrm{a}(2 \mathrm{n}-1) / 2\right]$} & {$\left[\mathrm{x}_{2}, \mathrm{y}_{1}+\mathrm{a}(2 \mathrm{n}-1) / 2\right]$} \\
\hline & (x) & $\begin{array}{l}\left(x_{2}, y_{1}+3 a / 2\right) \\
\left(x_{2}, y_{1}+a / 2\right) \\
\frac{\left(x_{2}, y_{1}\right)}{x}\end{array}$ \\
\hline
\end{tabular}

Fig. 3: The Motion Trajectory of the Spray Gun

2) The motion trajectory of the spray gun is $S$ shape, which is, the processing paths of the spraying device moving from one side of the work-piece to the other rectilinear. It is a cyclic process that does translation upwards firstly and then pulls back. The distance between the two adjacent paths is equal. Due to the possibility of coating obtained in the edge of work-piece is smaller than in the middle part, and the possibility of coating obtained in the middle area of spraying is bigger than both sides in the actual spraying work, so, in order to ensure the good spraying quality, during the spraying course, certain overlap is required on the two-time adjacent spraying paths[4]. Suppose the spray gun do translation downward by half of the spray band width, overlap the two-time adjacent spraying paths $\mathrm{a} / 2$. The motion path of the spray gun is shown in fig. 4 , the total length of this path is: $L=(n+1) 1+n \times a / 2$. In this formula, $n$ is the spraying journey minus 1 , the distance between the adjacent spraying paths is $a / 2$. The distance between two adjacent spraying paths that overlapped is $\mathrm{d}$.

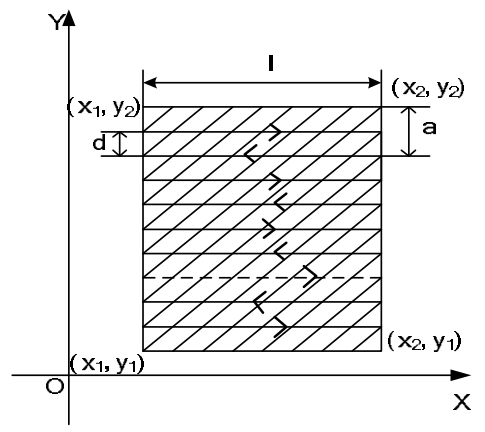

Fig. 4: The Motion Trajectory of the Spray Gun

Considering from all kinds of factors, the first motion trajectory for spraying work is comparatively simple, which only needs to complete easy rectilinear motion. However, this kind of spraying path has low work efficiency, meanwhile it is easy to produce gap between the two adjacent spraying paths, and the spraying thickness is uneven[5]. Compared with the first motion trajectory, the second one is higher in spraying efficiency, the spraying thickness to the work-piece 
to be machined is even and the product quality is improved. Therefore, this paper adopts the motion trajectory of the second spraying work.

Optimization of the motion trajectory of the spray gun. The objective of OTTP (optimal trajectory planning problems): find a trajectory that needs the shortest running distance during the processing course and takes the shortest spraying period[3]. Meanwhile, it carries out speed accelerating and reducing control towards the spraying device's linear motion downward. Before the spraying device starts to machine each plane, it controls the course nearby the starting point. Based on the above constraint conditions, it extends the path of the spray gun towards the surface of the work-piece to be machined for some distance, finally dispose the corner on each path into circular arc transition, for avoiding the vibration that may be caused from the sudden speed change of the spray gun and happened at the end of the robot, making the whole processing course under optimal implementation [6]. The motion trajectory of the spray gun after optimized as shown in fig.

5. Total length for this path is: $L=(n+1) 1+\pi \times n \times d / 2$.

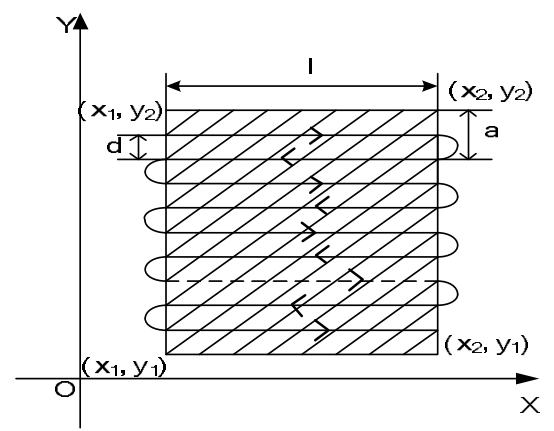

Fig. 5: The Motion Trajectory of the Spray Gun after Optimized

\section{Conclusion}

With the development of the industrial level in our country, the application of automatic spraying device is getting more and more widespread, and there is tremendous development whether in the study of the depth and breadth. How to apply for the intelligent algorithm to implement the spraying work on the complex surface becomes the completely meaningful research topic. Aiming at the mutual restriction between the running distance and the spraying time required by the spray gun on the plane, this paper put forward to a method about the trajectory planning and the optimization for the spray gun. By the time of meeting the shortest distance required of the spray gun, this method had saved the spraying time and improved the spraying efficiency.

\section{References}

[1] R. Gadow, A. Candel, M. Floristan: Surface and Coatings Technology. Vol. 205. (2010), pp. 1074-1079.

[2] H. S. Liu, X. B. Lai, W. X. Wu: Robotics and Computer Integrated Manufacturing. Vol. 29. (2014), pp. 309-317.

[3] J. Gregorya, A. Olivaresb, E. Staffettib: Systems \& Control Letters. Vol. 61. (2012), pp. 279-291.

[4] C. Rossi and S. Savino: Robotics and Computer Integrated Manufacturing. Vol. 29. (2013), pp. 139-156.

[5] M. R. Azizi and D. Nader: Mechanism and Machine Theory. Vol. 64. (2013), pp. 251-261.

[6] A. Gasparetto and V. Zanotto: Advances in Engineering Software. Vol. 41.(2010), pp. 548-556. 